\title{
Retraction Note: Exploring the ancestry differentiation and inference capacity of the 28-plex AISNPs
}

\author{
Wei-Qi Hao ${ }^{1,2}$. Jing Liu ${ }^{2}$ Li Jiang ${ }^{2}$ Jun-Ping Han ${ }^{2,3} \cdot$ Ling Wang $^{2} \cdot$ Jiu-Ling $\mathrm{Li}^{2} \cdot$ Quan $\mathrm{Ma}^{2} \cdot$ Chao Liu $^{1,4}$. \\ Hui-Jun Wang ${ }^{1} \cdot$ Cai-Xia Li ${ }^{2}$
}

Published online: 7 September 2021

๑) Springer-Verlag GmbH Germany, part of Springer Nature 2021

\section{Retraction Note to: International Journal of Legal Medi- cine (2019) 133:975-982}

https://doi.org/10.1007/s00414-018-1863-z

The Editors-in-Chief have retracted this article. Since publication, concerns were raised about the ethics and consent procedures for this study. We requested supporting documentation from the authors, including the application form submitted to the ethics committee and evidence of ethics approval. The documents supplied by the authors contain insufficient information related to the scope of the study for us to remain confident that the protocols complied with our editorial policies or are in line with international ethical standards [1].

Caixia Li stated on behalf of all co-authors that they do not agree to this retraction.

The original article can be found online at https://doi.org/10.1007/ s00414-018-1863-z.

Chao Liu

liuchaozf@163.com

$\bowtie$ Hui-Jun Wang

hjwang@smu.edu.cn

$\triangle$ Cai-Xia Li

licaixia@tsinghua.org.cn

1 Institute of Forensic Medicine, Southern Medical University, Guangzhou 510515, Guangdong, China

2 Beijing Engineering Research Center of Crime Scene Evidence Examination, National Engineering Laboratory for Forensic Science, Institute of Forensic Science, Beijing 100038, People's Republic of China

3 Technology Department of Chaoyang Sub-Bureau, Beijing Public Security Bureau, Beijing 100102, China

4 Guangdong Province Key Laboratory of Forensic Genetics, Guangzhou Forensic Science Institute, Guangzhou 510030, Guangdong, China

\section{Reference}

1. World Medical Association (2001) World Medical Association Declaration of Helsinki. Ethical principles for medical research involving human subjects. Bull World Health Organ 79(4):373

Publisher's note Springer Nature remains neutral with regard to jurisdictional claims in published maps and institutional affiliations. 